\title{
Review of: "Multiple groups of methanotrophic bacteria mediate methane oxidation in anoxic lake sediments"
}

\author{
Marcus Elvert ${ }^{1}$ \\ 1 Universität Bremen
}

Potential competing interests: The author(s) declared that no potential competing interests exist.

This submission by Su and co-workers explores the presence and activity of bacterial aerobic methanotrophs in lake sediments (here: Lake Sempach, Switzerland) under conditions of complete oxygen depletion. The hidden process of "aerobic methanotrophy" has been frequently reported from different lake environments using various approaches but the ultimate electron acceptor(s) has been rather elusive so far. Because the process represents an additional methane sink that is not fully accounted for it is important to study, but its identification is far from being trivial. Here, the authors have designed a study in which they nicely combine depth-dependent environmental data, including in situ radiotracer measurements (using ${ }^{14} \mathrm{CH}_{4}$ ), with those from time-dependent stable isotope probing incubation experiments (using $\left.{ }^{13} \mathrm{CH}_{4}\right)$ where they added probable electron acceptors $\left(\mathrm{O}_{2}, \mathrm{NO}_{3}{ }^{-} / \mathrm{NO}_{2}{ }^{-}\right.$, metal oxides, and $\mathrm{SO}_{4}{ }^{2-}$ ). In the incubation experiments, the process was monitored by methane turnover products, and bacterial fatty acid and gene sequence analyses, the latter two after prolonged time of incubation. At the end, the authors derive at the conclusion that different groups of facultative aerobic methanotrophs are playing a role but no single electron acceptor could be favored. However, the methane turnover potential was highest by adding oxygen, which may relate to the presence of bacterial methanotrophic clades in the uppermost sediments triggered by sporadic oxygen supply caused by the artificial aeration activity running over the last decades in the lake (is it sporadic?).

I just have a few, mostly editorial remarks the authors should consider.

Materials and Methods:

It would have been perfect to analyze the environmental distribution of bacterial fatty acids (as a natural endmember) next to DNA. Why was this omitted?

I am heavily missing information on the gas chromatographic conditions (injection mode, T-program, column used, sometimes carrier gas as well) applied for the measurement of gases and lipid fractions. Please add this. References would also help. 
References are likewise needed for the ICP-OES measurements, DIC determinations and the use of microelectrode sensors. How have those measurements being calibrated?

For lipid identification and isotope determination, the reader is in need of how double bond isomers had been determined. All of them are likely not present in the standard mixtures used and cannot be clearly identified via GC-MS. The same accounts for standardization and precision of $d^{13} \mathrm{C}$-values, especially considering the highly ${ }^{13} \mathrm{C}$-enriched ones (how many numbers of significant figures are actually justified?). Please also provide more information (or a reference) on how the correction of introduced carbon had been done.

Results:

For better orientation, please relate all results to each figure panel in Figs. 1, 3 and 4 (to be added, see below), and do not, for example, start with Fig. 1B before not mentioning Fig. 1A.

For Fig. 1K, I urge the authors to prepare it as a single figure. Details are much too small now.

Mention the fact that a doubling in rates is seen between 30 and 60 days in Fig. 2.

\section{Discussion:}

I would generally favor to see some of this text being separated into subsections with headers. Otherwise, this long text is hard to digest.

Line 409: It is not fully getting clear if the aeration is performed in a sporadic or continuous mode. This would have an effect on whether the oxygen profile is representing a snap shot or not.

Line 416: SMTZs are found very close to the surface at cold methane seeps. This should be mentioned.

Line 448: I do not think the authors can state that there is growth. Fatty acids are not increasing and no other biomass indicators are shown. There is a change in relative abundance of detected genes and active C-incorporation into fatty acids, I agree.

Line 456: Methane incorporation into these fatty acids was similarly observed by Bar-Or et al. (2017; doi: 10.1021/acs.est.7b03126) during anaerobic incubation studies using iron oxides as electron acceptors. Type I methanotrophs were involved ( $1 \%$ abundance of gammaproteobacteria in the original anoxic sediment horizons, see Bar-Or et al., 2015; doi: 10.5194/bg-12-2847-2015) and pmoA genes were increased during incubation. 
Line 478: In order to justify this argument, I would have thought that nitrate additions would boost their numbers and, in turn, methane turnover. I do not see this and the authors themselves falsify this in the next paragraph.

Line 495: Please add the Bar-Or et al. (2017) study which showed similar results.

Line 498: This argumentation is at odds with the positive results reported a few lines before. What was different in those studies that metal oxides stimulated methane turnover?

Line 523: Or another not-yet-identified pathway?

Line 535: Good point but please check Vigderovich et al. (2021, doi: 10.5194/bg-2021-223) in Biogeosciences Discussion on this.

Line 542: This section would benefit from the results presented in Valenzuela et al. (2019; doi: 10.1016/j.scitotenv.2018.09.388) who exactly provided evidence for this in a tropical wetland.

Figure 4: Please label these panels with letters as done for Figs. 1 and 3. 